\title{
Convergence behavior of single-step GBLUP and SNPBLUP for different termination criteria
}

\author{
Jeremie Vandenplas ${ }^{1 *} \mathbb{D}$, Mario P. L. Calus ${ }^{1}$, Herwin Eding ${ }^{2}$, Mathijs van Pelt ${ }^{2}$, Rob Bergsma $^{3}$ and Cornelis Vuik ${ }^{4}$
}

\begin{abstract}
Background: The preconditioned conjugate gradient (PCG) method is the current method of choice for iterative solving of genetic evaluations. The relative difference between two successive iterates and the relative residual of the system of equations are usually chosen as a termination criterion for the PCG method in animal breeding. However, our initial analyses showed that these two commonly used termination criteria may report that a PCG method applied to a single-step single nucleotide polymorphism best linear unbiased prediction (ssSNPBLUP) is not converged yet, whereas the solutions are accurate enough for practical use. Therefore, the aim of this study was to propose two termination criteria that have been (partly) developed in other fields, but are new in animal breeding, and to compare their behavior to that of the two termination criteria widely used in animal breeding for the PCG method applied to ssSNPBLUP. The convergence patterns of ssSNPBLUP were also compared to the convergence patterns of single-step genomic BLUP (ssGBLUP).

Results: Building upon previous work, we propose two termination criteria that take the properties of the system of equations into account. These two termination criteria are directly related to the relative error of the iterates with respect to the true solutions. Based on pig and dairy cattle datasets, we show that the preconditioned coefficient matrices of sSSNPBLUP and SSGBLUP have similar properties when using a second-level preconditioner for ssSNPBLUP. Therefore, the PCG method applied to ssSNPBLUP and ssGBLUP converged similarly based on the relative error of the iterates with respect to the true solutions. This similar convergence behavior between ssSNPBLUP and ssGBLUP was observed for both proposed termination criteria. This was, however, not the case for the termination criterion defined as the relative residual when applied to the dairy cattle evaluations.

Conclusion: Our results showed that the PCG method can converge similarly when applied to sSSNPBLUP and to ssGBLUP. The two proposed termination criteria always depicted these similar convergence behaviors, and we recommend them for comparing convergence properties of different models and for routine evaluations.
\end{abstract}

\section{Background}

The current method of choice for genomic evaluations is the so-called single-step genomic best linear unbiased prediction (ssGBLUP) that simultaneously analyses phenotypic and pedigree information of genotyped and non-genotyped animals with genomic information of

*Correspondence: jeremie.vandenplas@wur.nl

${ }^{1}$ Animal Breeding and Genomics, Wageningen UR, P.O. Box 338, 6700 AH Wageningen, The Netherlands

Full list of author information is available at the end of the article genotyped animals [1]. The ssGBLUP model considers genomic information by combining genomic and pedigree relationships into a combined genomic-pedigree relationship matrix [2,3]. Equivalent models that directly estimate SNP effects and that do not rely on the genomic relationship matrix $\mathbf{G}$, hereafter called ssSNPBLUP, were also proposed [4-6].

The solving method of choice in the last two decades for breeding value estimation models is the preconditioned conjugate gradient (PCG) method with a traditional (block-)diagonal preconditioner [7]. However,

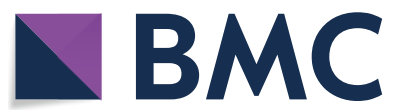

(c) The Author(s) 2021. This article is licensed under a Creative Commons Attribution 4.0 International License, which permits use, sharing, adaptation, distribution and reproduction in any medium or format, as long as you give appropriate credit to the original author(s) and the source, provide a link to the Creative Commons licence, and indicate if changes were made. The images or other third party material in this article are included in the article's Creative Commons licence, unless indicated otherwise in a credit line to the material. If material is not included in the article's Creative Commons licence and your intended use is not permitted by statutory regulation or exceeds the permitted use, you will need to obtain permission directly from the copyright holder. To view a copy of this licence, visit http://creativeco mmons.org/licenses/by/4.0/. The Creative Commons Public Domain Dedication waiver (http://creativecommons.org/publicdomain/ zero/1.0/) applies to the data made available in this article, unless otherwise stated in a credit line to the data. 
when applied to ssSNPBLUP, it results in poor convergence $[8,9]$. We have previously shown that using a second-level preconditioner improves the PCG convergence rate considerably $[9,10]$.

An important parameter of an iterative solver is its termination criterion. A termination criterion should be defined to stop the iterative process at an appropriate level of convergence. The relative difference between two successive iterates and the relative residual of the system of equations is often chosen as a termination criterion for the PCG method in animal breeding [7, 11-13]. However, our initial analyses showed that these two commonly used termination criteria may report that a PCG
ssGBLUP

For ssGBLUP [1], the vector $\mathbf{b}_{G}$ is equal to $\mathbf{b}_{G}=\left[\begin{array}{c}\mathbf{X}^{\prime} \mathbf{R}^{-1} \mathbf{y} \\ \mathbf{W}_{n}^{\prime} \mathbf{R}_{n}^{-1} \mathbf{y}_{n} \\ \mathbf{W}_{g}^{\prime} \mathbf{R}_{g}^{-1} \mathbf{y}_{g}\end{array}\right]$ where the subscripts $g$ and $n$ refer to $n_{g}$ genotyped and $n_{n}$ non-genotyped animals, respectively, $\mathbf{y}$ is the vector of records, and the matrices $\mathbf{X}, \mathbf{W}_{n}$ and $\mathbf{W}_{g}$ are incidence matrices relating records to the corresponding effects, and the matrix $\mathbf{R}^{-1}=\left[\begin{array}{cc}\mathbf{R}_{n}^{-1} & \mathbf{0} \\ \mathbf{0} & \mathbf{R}_{g}^{-1}\end{array}\right]$ is the inverse of the residual (co)variance structure matrix.

The coefficient matrix $\mathbf{C}_{G}$ is equal to:

$$
\mathbf{C}_{G}=\left[\begin{array}{ccc}
\mathbf{X}^{\prime} \mathbf{R}^{-1} \mathbf{X} & \mathbf{X}_{n}^{\prime} \mathbf{R}_{n}^{-1} \mathbf{W}_{n} & \mathbf{X}_{g}^{\prime} \mathbf{R}_{g}^{-1} \mathbf{W}_{g} \\
\mathbf{W}_{n}^{\prime} \mathbf{R}_{n}^{-1} \mathbf{X}_{n} & \mathbf{W}_{n}^{\prime} \mathbf{R}_{n}^{-1} \mathbf{W}_{n}+\mathbf{A}^{n n} \sigma_{u}^{-2} & \mathbf{A}^{n g} \sigma_{u}^{-2} \\
\mathbf{W}_{g}^{\prime} \mathbf{R}_{g}^{-1} \mathbf{X}_{g} & \mathbf{A}^{g n} \sigma_{u}^{-2} & \mathbf{W}_{g}^{\prime} \mathbf{R}_{g}^{-1} \mathbf{W}_{g}+\left(\mathbf{A}^{g g}+\mathbf{G}^{-1}-\mathbf{A}_{g g}^{-1}\right) \sigma_{u}^{-2}
\end{array}\right]
$$

method applied to ssSNPBLUP is not converged yet, whereas the solutions are accurate enough for practical use. Therefore, the aim of this study was to implement termination criteria that have been (partly) developed in other fields, but are new in animal breeding, and to compare their behaviors to that of different termination criteria that are widely used in animal breeding for the PCG method applied to ssSNPBLUP. A comparison of the convergence patterns of ssSNPBLUP and of ssGBLUP was also performed.

\section{Methods}

\section{Single-step genomic evaluations}

In this study, we investigate the convergence behavior of the PCG method applied to ssGBLUP $[2,14]$ and to the ssSNPBLUP linear equations system proposed by Gengler et al. [15] and by Liu et al. [6]. The two systems of equations can be summarized as:

$$
\mathbf{C}_{j} \mathbf{x}_{j}=\mathbf{b}_{j},
$$

where $j$ refers to ssGBLUP $(j=\mathrm{G})$, or to the ssSNPBLUP proposed by Liu et al. [6] ( $j=\mathrm{L}), \mathbf{C}_{j}$ is a symmetric (semi-) definite coefficient matrix, $\mathbf{x}_{j}$ is the vector of solutions, and $\mathbf{b}_{j}$ is the right-hand side of the linear system.

For simplicity, and without loss of generality, the different matrices and vectors for ssGBLUP and ssSNPBLUP are described below for an univariate animal model. where $\sigma_{u}^{-2}$ is the inverse of the additive genetic variance, $\mathbf{A}^{-1}=\left[\begin{array}{ll}\mathbf{A}^{n n} & \mathbf{A}^{n g} \\ \mathbf{A}^{g n} & \mathbf{A}^{g g}\end{array}\right]$ is the inverse of the pedigree relationship matrix, $\mathbf{A}_{g g}$ is the pedigree relationship between genotyped animals, and $\mathbf{G}=\frac{1-w}{m} \mathbf{Z} \mathbf{Z}^{\prime}+w \mathbf{A}_{g g}$ is the genomic relationship matrix with $w$ being the proportion of variance (due to additive genetic effects) considered as residual polygenic effects, and $m=2 \sum p_{o}\left(1-p_{o}\right)$ with $p_{o}$ being the allele frequency of the oth SNP. The matrix $\mathbf{Z}$ contains the SNP genotypes (coded as 0 for one homozygous genotype, 1 for the heterozygous genotype, or 2 for the alternate homozygous genotype) centered by their observed means.

The vector of solutions is equal to $\mathbf{x}_{G}=\left[\begin{array}{c}\hat{\boldsymbol{\beta}} \\ \hat{\mathbf{u}}_{n} \\ \hat{\mathbf{u}}_{g}\end{array}\right]$, where $\boldsymbol{\beta}$ is the vector of fixed effects, $\mathbf{u}_{n}$ is the vector of additive genetic effects for non-genotyped animals, and $\mathbf{u}_{g}$ is the vector of additive genetic effects for genotyped animals.

\section{SSSNPBLUP}

For ssSNPBLUP [6], the vector $\mathbf{b}_{L}$ is equal to $\mathbf{b}_{L}=\left[\begin{array}{c}\mathbf{X}^{\prime} \mathbf{R}^{-1} \mathbf{y} \\ \mathbf{W}_{n}^{\prime} \mathbf{R}_{n}^{-1} \mathbf{y}_{n} \\ \mathbf{W}_{g}^{\prime} \mathbf{R}_{g}^{-1} \mathbf{y}_{g} \\ \mathbf{0}\end{array}\right]$.

The coefficient matrix $\mathbf{C}_{L}$ is equal to:

$$
\mathbf{C}_{L}=\left[\begin{array}{cccc}
\mathbf{X}^{\prime} \mathbf{R}^{-1} \mathbf{X} & \mathbf{X}_{n}^{\prime} \mathbf{R}_{n}^{-1} \mathbf{W}_{n} & \mathbf{X}_{g}^{\prime} \mathbf{R}_{g}^{-1} \mathbf{W}_{g} & \mathbf{0} \\
\mathbf{W}_{n}^{\prime} \mathbf{R}_{n}^{-1} \mathbf{X}_{n} & \mathbf{W}_{n}^{\prime} \mathbf{R}_{n}^{-1} \mathbf{W}_{n}+\mathbf{A}^{n n} \sigma_{u}^{-2} & \mathbf{A}^{n g} \sigma_{u}^{-2} & \mathbf{0} \\
\mathbf{W}_{g}^{\prime} \mathbf{R}_{g}^{-1} \mathbf{X}_{g} & \mathbf{A}^{g n} \sigma_{u}^{-2} & \mathbf{W}_{g}^{\prime} \mathbf{R}_{g}^{-1} \mathbf{W}_{g}+\left(\mathbf{A}^{g g}+\mathbf{\Sigma}_{L, 11}\right) \sigma_{u}^{-2} & \mathbf{\Sigma}_{L, 12} \sigma_{u}^{-2} \\
\mathbf{0} & \mathbf{0} & \boldsymbol{\Sigma}_{L, 21} \sigma_{u}^{-2} & \mathbf{\Sigma}_{L, 22} \sigma_{u}^{-2}
\end{array}\right]
$$


where $\boldsymbol{\Sigma}_{L}=\left[\begin{array}{ll}\boldsymbol{\Sigma}_{L, 11} & \boldsymbol{\Sigma}_{L, 12} \\ \boldsymbol{\Sigma}_{L, 21} & \boldsymbol{\Sigma}_{L, 22}\end{array}\right]=\left[\begin{array}{cc}\left(\frac{1}{w}-1\right) \mathbf{A}_{g g}^{-1} & -\frac{1}{w} \mathbf{A}_{g g}^{-1} \mathbf{Z} \\ -\frac{1}{w} \mathbf{Z}^{\prime} \mathbf{A}_{g g}^{-1} & \frac{1}{w} \mathbf{Z}^{\prime} \mathbf{A}_{g g}^{-1} \mathbf{Z}+\frac{m}{1-w} \mathbf{I}\end{array}\right]$.

The vector $\mathbf{x}_{L}$ is equal to $\mathbf{x}_{L}=\left[\begin{array}{c}\hat{\boldsymbol{\beta}} \\ \hat{\mathbf{u}}_{n} \\ \hat{\mathbf{u}}_{g} \\ \hat{\mathbf{g}}\end{array}\right]$ where $\mathbf{g}$ is the vector of SNP effects.

The equivalence between ssGBLUP and ssSNPBLUP can be shown by absorbing the equations of the SNP effects of ssSNPBLUP and by using the Woodbury matrix identity [16]:

$$
\begin{aligned}
\mathbf{G}^{-1}-\mathbf{A}_{g g}^{-1} & =\left(\frac{1-w}{m} \mathbf{Z} \mathbf{Z}^{\prime}+w \mathbf{A}_{g g}\right)^{-1}-\mathbf{A}_{g g}^{-1} \\
& =\left(\frac{1}{w}-1\right) \mathbf{A}_{g g}^{-1}-\frac{1}{w} \mathbf{A}_{g g}^{-1} \mathbf{Z}\left(\frac{1}{w} \mathbf{Z}^{\prime} \mathbf{A}_{g g}^{-1} \mathbf{Z}+\frac{m}{1-w} \mathbf{I}\right)^{-1} \mathbf{Z}^{\prime} \mathbf{A}_{g g}^{-1} \frac{1}{w}
\end{aligned}
$$

invariant, and are therefore usually preferred over absolute termination criteria.

In animal breeding, the PCG method is often stopped when the relative residual (denoted by $C R$ ) is lower than or equal to a pre-defined threshold $\epsilon_{C R}$, that is $[11,12]$ :

$$
\frac{\left\|\mathbf{r}_{i}\right\|}{\|\mathbf{b}\|} \leq \epsilon_{C R}
$$

where $\mathbf{r}_{i}=\mathbf{b}-\mathbf{C} \hat{\mathbf{x}}_{i}$.

It can be shown that the termination criterion $C R$ is related to the relative error in $\mathbf{x}, e_{r, i}$ (Eq. 2), as follows [19]

\section{PCG method}

A PCG method is an iterative method that uses successive approximations to obtain more accurate solutions for a linear system at each iteration step [17]. In our implementation, the preconditioned system of linear equations of ssGBLUP and of ssSNPBLUP required by the PCG method has the form (with subscripts omitted):

$$
\tilde{\mathbf{M}}^{-1} \mathbf{C x}=\tilde{\mathbf{M}}^{-1} \mathbf{b},
$$

where $\tilde{\mathbf{M}}=\mathbf{D M}$ with $\mathbf{M}$ being a (block-)diagonal preconditioner defined below separately for each analysis, and D being an identity matrix for ssGBLUP, or a second-level diagonal preconditioner for SSSNPBLUP as proposed by Vandenplas et al. [10].

\section{Termination criteria}

The relative error in $\mathbf{x}$ at the $i$-th iteration of the PCG method is defined as:

$$
e_{r, i}=\frac{\left\|\mathbf{x}-\hat{\mathbf{x}}_{i}\right\|}{\|\mathbf{x}\|}
$$

where $\hat{\mathbf{x}}_{i}$ is an approximate solution of $\mathbf{x}$ at the $i$-th iteration and $\|$.$\| is the 2$-norm.

Unfortunately, because the true solution $\mathbf{x}$ is unknown, the relative error in $\mathbf{x}\left(e_{r, i}\right)$ cannot be computed and used as termination criterion. Therefore, alternative termination criteria must be used. A good termination criterion is important for iterative solvers, and should identify when $e_{r, i}$ is small enough to stop the iterative process. If this is not the case, the iterative process might be stopped too soon, resulting in useless approximate solutions, or take an unnecessary long time or never stop [18]. Relative termination criteria as those proposed below are scaling (see Additional file 1 for a derivation):

$$
e_{r, i} \leq \kappa(\mathbf{C}) \frac{\left\|\mathbf{r}_{i}\right\|}{\|\mathbf{b}\|}
$$

where $\kappa(\mathbf{C})$ is the effective spectral condition number of $\mathrm{C}$ defined as the ratio of its largest to smallest positive eigenvalues [20].

There are several drawbacks of the termination criterion CR (Eq. 3). First, the termination criterion CR may be difficult to satisfy when $\mathbf{C}$ is very ill-conditioned, and thus when $\kappa(\mathbf{C})$ is large [18]. Second, the comparison of the convergence rates of the PCG method applied to different systems of equations may lead to wrong conclusions if $\kappa(\mathbf{C})$ associated with the compared systems are very different. Indeed, using the same threshold $\epsilon_{C R}$ for the compared systems would result in PCG methods stopping at a same level of CR but at different levels of $e_{r}$. Finally, it is worth noting that $\kappa(\mathbf{C})$ could be estimated at low costs during an unpreconditioned conjugate gradient (CG) process based on the equivalence of the CG and Lanczos methods [18, 21]. However, this study focuses on PCG methods for solving preconditioned systems of equations. In this context, the correction of the termination criterion CR by $\kappa(\mathbf{C})$ cannot be applied because $\kappa(\mathbf{C})$ cannot be computed easily for most large systems of equations.

Another termination criterion that is often used in animal breeding, is the relative difference between two consecutive iterates (denoted by CD) of the PCG method, that is [11]:

$$
\frac{\left\|\hat{\mathbf{x}}_{i-1}-\hat{\mathbf{x}}_{i}\right\|}{\left\|\hat{\mathbf{x}}_{i}\right\|} \leq \epsilon_{C D}
$$

A drawback of the termination criterion CD is that it is not related to the relative error in $\mathbf{x}, e_{r, i}$. Therefore the 
termination criterion $\mathrm{CD}$ could be satisfied while $e_{r, i}$ is still large.

A third termination criterion (denoted by CK) is defined as follows [21, 22]:

$$
\frac{1}{\mu_{1}} \frac{\left\|\tilde{\mathbf{M}}^{-1} \mathbf{r}_{i}\right\|}{\left\|\hat{\mathbf{x}}_{i}\right\|} \leq \epsilon_{C K}
$$

where $\mu_{1}$ is the smallest active eigenvalue (i.e. the smallest positive eigenvalue that influences the convergence [23]) of $\tilde{\mathbf{M}}^{-1} \mathbf{C}$.

It can be shown that the termination criterion $\mathrm{CK}$ is related to the relative error in $\mathbf{x}, e_{r, i}$ (Eq. 2), as follows [21, 22]:

$$
e_{r, i} \leq \frac{1}{\mu_{1}} \frac{\left\|\tilde{\mathbf{M}}^{-1} \mathbf{r}_{i}\right\|}{\left\|\hat{\mathbf{x}}_{i}\right\|} .
$$

Therefore, this termination criterion CK allows the user to specify the desired relative accuracy $\epsilon_{C K}$ in the computed solution $\hat{\mathbf{x}}_{i}$. To our knowledge, the termination criterion CK was never applied in animal breeding, and is scarcely used in other fields.

Finally, we introduce a fourth termination criterion (denoted by CM) defined as follows (see Additional file 2 for a derivation):

$$
\kappa\left(\tilde{\mathbf{M}}^{-1} \mathbf{C}\right) \frac{\left\|\tilde{\mathbf{M}}^{-1} \mathbf{r}_{i}\right\|}{\left\|\tilde{\mathbf{M}}^{-1} \mathbf{b}\right\|} \leq \epsilon_{C M},
$$

where $\kappa\left(\tilde{\mathbf{M}}^{-1} \mathbf{C}\right)$ is the effective spectral condition number of $\tilde{\mathbf{M}}^{-1} \mathbf{C}$.

It can be shown that the termination criterion $\mathrm{CM}$ is related to the relative error in $\mathbf{x}, e_{r, i}$ (Eq. 2), as follows (see Additional file 2 for a derivation):

$$
e_{r, i} \leq \kappa\left(\tilde{\mathbf{M}}^{-1} \mathbf{C}\right) \frac{\left\|\tilde{\mathbf{M}}^{-1} \mathbf{r}_{i}\right\|}{\left\|\tilde{\mathbf{M}}^{-1} \mathbf{b}\right\|} .
$$

Like the termination criterion $\mathrm{CK}$, the termination criterion $\mathrm{CM}$ is defined at the scale of the relative accuracy in $\mathbf{x}$. Thus, the termination criterion CM allows the user to specify the desired relative accuracy in $\mathbf{x}$. The termination criterion $\mathrm{CM}$ can be also considered as being the termination criterion $\mathrm{CR}$ applied to the preconditioned system of equations, instead of the system of equations directly (1). Finally, it is worth noting that the termination criterion $\mathrm{CM}$ is equal to the relative error in $\mathbf{x}$ if $\tilde{\mathbf{M}}=\mathbf{C}$.

The termination criteria $\mathrm{CK}$ and $\mathrm{CM}$ require $\mu_{1}$ and $\kappa\left(\tilde{\mathbf{M}}^{-1} \mathbf{C}\right)$ for their computation. The estimates of $\mu_{1}$ and of $\kappa\left(\tilde{\mathbf{M}}^{-1} \mathbf{C}\right)$ can be obtained at low costs during the PCG process using the Lanczos method based on information obtained from the PCG method, e.g., as proposed by Kaasschieter [21] or as described below.

\section{Relationships between termination criteria applied to ssGBLUP and ssSNPBLUP}

When comparing the termination criterion CR between ssGBLUP and ssSNPBLUP, it is worth noting that $\left\|\mathbf{b}_{G}\right\|=\left\|\mathbf{b}_{L}\right\|$ because all entries of the right-hand sides are the same, except for the entries corresponding to the SNP equations that are equal to 0 . Therefore, when estimates for the common entries between $\hat{\mathbf{x}}_{G}$ and $\hat{\mathbf{x}}_{L}$ are equal (i.e., when $\hat{\boldsymbol{\beta}}_{G}=\hat{\boldsymbol{\beta}}_{L}, \hat{\mathbf{u}}_{n, G}=\hat{\mathbf{u}}_{n, L}$, and $\hat{\mathbf{u}}_{g, G}=\hat{\mathbf{u}}_{g, L}$ ), any observed differences between CR for ssGBLUP and for sSSNPBLUP are a consequence of the presence of the estimates of SNP effects in the solution vector of ssSNPBLUP $\hat{\mathbf{x}}_{L}$. More specifically, it can be shown in this case that the differences between CR for ssGBLUP and for ssSNPBLUP can be explained by the error due to the PCG iterative process in estimating the SNP effects $\hat{\mathbf{g}}, \epsilon_{L}$ : $\epsilon_{L}=\hat{\mathbf{g}}_{L}-\left(\frac{1}{w} \mathbf{Z}^{\prime} \mathbf{A}_{g g}^{-1} \mathbf{Z}+\frac{m}{1-w} \mathbf{I}\right)^{-1} \frac{1}{w} \mathbf{A}_{g g}^{-1} \mathbf{Z} \hat{\mathbf{u}}_{g, L}$.

Indeed, after some derivations (see Additional file 3 for details), we can show that:

$$
\left\|\mathbf{r}_{L}\right\|=\left\|\left[\begin{array}{c}
\mathbf{r}_{G} \\
\mathbf{0}
\end{array}\right]+\left[\begin{array}{c}
\mathbf{0} \\
\frac{1}{w} \mathbf{A}_{g g}^{-1} \mathbf{Z} \sigma_{u}^{-2} \epsilon_{L}
\end{array}\right]\right\|,
$$

where $\mathbf{r}_{L}=\mathbf{b}_{L}-\mathbf{C}_{L} \hat{\mathbf{x}}_{L}$ is the residual of the ssSNPBLUP system of equations, and $\mathbf{r}_{G}=\mathbf{b}_{G}-\mathbf{C}_{G} \hat{\mathbf{x}}_{G}$ is the residual of the ssGBLUP system of equations.

Finally, $\left\|\mathbf{x}_{G}\right\|$ is approximately equal to $\left\|\mathbf{x}_{L}\right\|$ (similarly for $\left\|\hat{\mathbf{x}}_{G}\right\|$ and $\left.\left\|\hat{\mathbf{x}}_{L}\right\|\right)$ when the common entries of both vectors are equal. Indeed, the only different entries between these two vectors are the additional estimates of SNP effects that are of several thousand orders of magnitude lower than solutions of other fixed and random effects (e.g., genetic additive effects). Furthermore, the number of SNP effects (e.g., 50,000) is relatively low in comparison to the total number of equations in evaluations with a deep pedigree (e.g., with millions of animals). Therefore, the termination criterion $e_{r}$ for ssGBLUP and for ssSNPBLUP should have similar values, provided that the common solutions of both systems are similar.

\section{Data}

The first data set used in this study, hereafter referred to as the FIN data set, was provided by Topigs Norsvin (The Netherlands). The other two data sets used in this study, hereafter referred to as KAR and LON data sets, were provided by CRV BV (The Netherlands). 
The FIN data set and associated variance components were extracted from an 11-trait genetic evaluation for grower-finisher traits. After extraction, the data file included 752,067 records with a single record per animal (all born before July 2017), and all records had missing observations for at least one trait. The pedigree included 835,562 animals. The genotypes included 23,102 segregating SNPs, and were associated with 38,488 animals. The 11-trait mixed model included random effects (litter, pen, group, additive genetic and residual), fixed covariables [weight at start, at halfway, and at the end of the feed intake trajectory, weight (linear and quadratic) nested within farm-line-sex, probability of the sire passing the favorable allele for insulin-growth factor 2 (IGF2)] and fixed cross-classified effects (trial, farm-line-sex, compartment within farm). The traits included average daily gain measured across two time periods, back fat, loin depth, and feed intake across the entire testing period (all as separate traits for purebred and crossbred animals), and feed intake across the second half of the testing period, for purebred animals only.

The KAR data set and associated variance components were from the four-trait routine genetic evaluation of December 2019 for temperament and milking speed of dairy cattle for the Netherlands and the Flemish region of Belgium [24, 25]. Performances in each of these two countries were considered as different traits. The data file included 4,058,154 records with a single record per animal. The pedigree included $6,344,482$ animals. The genotypes included 37,995 segregating SNPs, and were associated with 123,644 animals. The four-trait mixed model included random effects (additive genetic and residual), fixed co-variables (heterosis and recombination) and fixed cross-classified effects (herd $\times$ year $\times$ season at classification, age at classification, lactation stage at classification, milk yield and month of calving) [24, 25].

The LON data set and associated variance components were from the univariate routine genetic evaluation of August 2019 for longevity of dairy cattle for the Netherlands and the Flemish region of Belgium [26, 27]. The data file included $408,107,042$ records associated with $12,528,520$ animals. The pedigree included $14,589,796$ animals. The genotypes included 37,995 segregating SNPs, and were associated with 120,000 animals that were randomly selected among 192,714 genotyped animals. The univariate random regression mixed model included random effects (Legendre polynomials (5th order) and residual), fixed co-variables (heterosis and recombination) and fixed cross-classified effects (herd $\times$ year $\times$ season $\times$ lactation stage, year $\times$ season $\times$ age of first calving $\times$ within-herd production level $\times$ lactation-stage, herd size change) $[26,27]$.

\section{Analyses}

The ssGBLUP and ssSNPBLUP systems for the three data sets were solved using a Fortran 2003 program that is described in Vandenplas et al. [28]. All real vectors and matrices (including the preconditioner) were stored inmemory using double-precision real arrays.

For the FIN data set, the preconditioner $\mathbf{M}$ had a blockdiagonal structure that included all equations for the fixed effects, and a block-diagonal structure for the random effects with blocks corresponding to equations for all traits within a level (e.g., an animal). For the KAR data set, the preconditioner $\mathbf{M}$ included only the diagonal elements of the coefficient matrix for the fixed effects, and a block-diagonal structure for the random effects with blocks corresponding to equations for different traits within a level (see [9] for more details). For the LON data set, two different preconditioners were tested to evaluate their impact on convergence. In the first case, the preconditioner $\mathbf{M}$ had a diagonal structure for all effects. Hereafter we refer to this evaluation as the LON evaluation. In the second case, the preconditioner $\mathbf{M}$ had a diagonal structure for the fixed effects, and blocks of elements for the random effects with blocks corresponding to equations for the different Legendre polynomials within a level (i.e. an animal). Hereafter we refer to this evaluation as the LON + block evaluation. For the three data sets, the proportion of variance (due to additive genetic effects) considered as residual polygenic effects, $w$, was assumed to be equal to 0.10 or 0.30 to evaluate its impact on convergence.

For analyzing the different termination criteria, all systems of equations were solved twice with the PCG method. All PCG processes iterated until the termination criterion $\mathrm{CM}$ or $\mathrm{CK}$ was $\leq 5 . * 10^{-3}$, ensuring a relative error in the solution $\leq 5 . * 10^{-3}$. Each solution vector obtained from the first iterative process, hereafter called manufactured solution, was pre-multiplied by the corresponding coefficient matrix to obtain a manufactured right-hand side. The systems of equations were then solved a second time after replacing the right-hand sides computed from the datasets by the manufactured right-hand sides. The manufactured solutions were therefore the true solutions of these systems of equations with manufactured right-hand sides. All results presented in this study are related to these systems of equations.

The smallest and largest eigenvalues that influence the convergence were approximated by the smallest and largest Ritz values, respectively. These extremal Ritz values were obtained every 200 -th iteration, starting at iteration 200, using the Lanczos method based on information obtained from the PCG method [21]. In our approach, the main computational cost consisted of the 

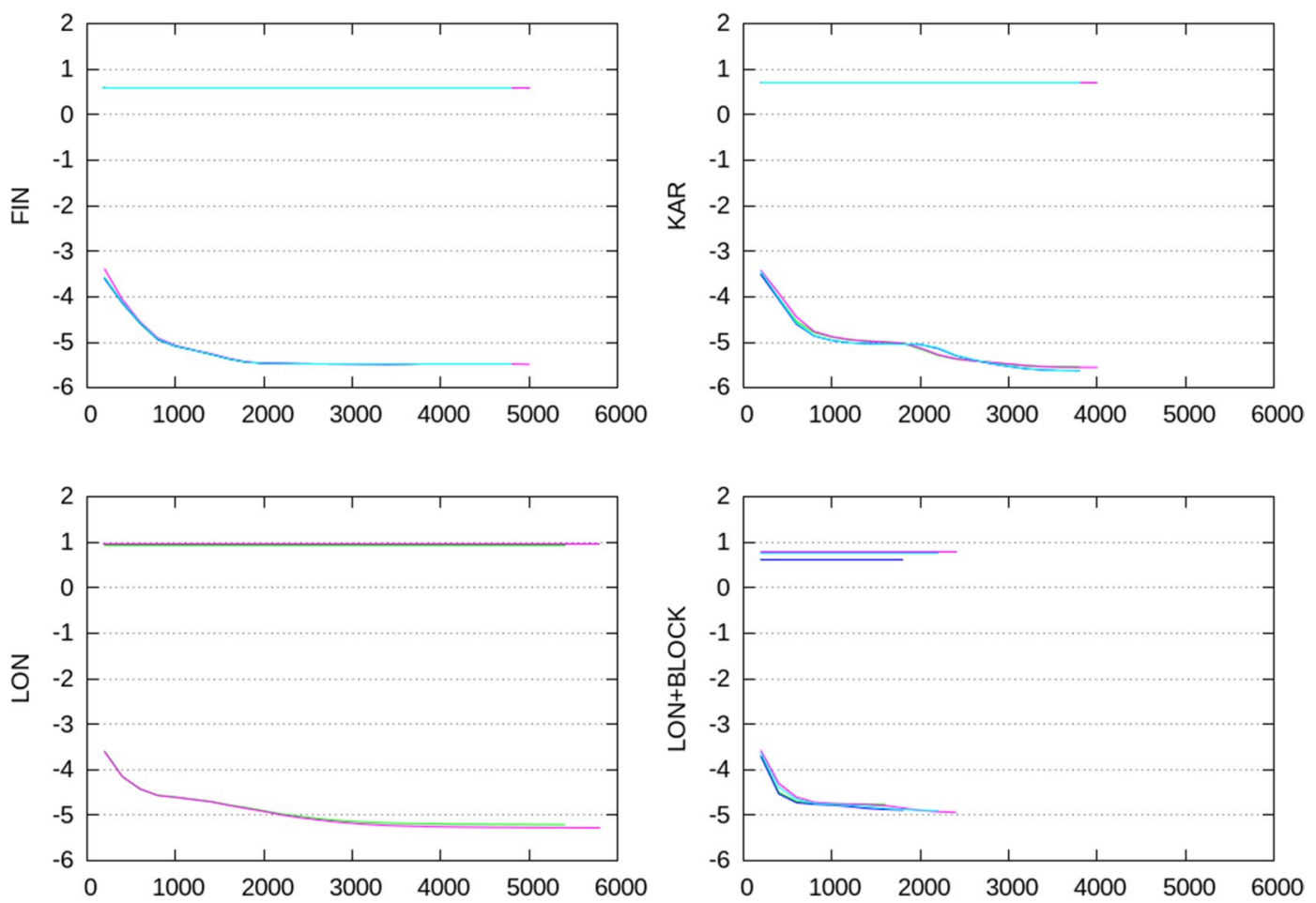

ssGBLUP(10)

ssGBLUP(30)

sSSNPBLUP(10)

SSSNPBLUP(30)

Fig. 1 Logarithm of the smallest and largest Ritz values (on the $y$-axis) for the different evaluations. Smallest Ritz values are depicted for ssGBLUP with a proportion of residual polygenic variance equal to 10\%, for ssGBLUP with a proportion of residual polygenic variance equal to $30 \%$, for ssSNPBLUP with a proportion of residual polygenic variance equal to 10\%, and sSSNPBLUP with a proportion of residual polygenic variance equal to $30 \%$

Table 1 Characteristics of systems for ssGBLUP and ssSNPBLUP

\begin{tabular}{|c|c|c|c|c|c|c|}
\hline Evaluation & Model $^{a}$ & \#Equations & \#lterations & Smallest eig. & Largest eig. & $\kappa^{\mathrm{b}}$ \\
\hline \multirow[t]{4}{*}{ FIN } & ssGBLUP (10) & $11,373,208$ & 4912 & $3.270 * 10^{-6}$ & 3.924 & $1.200 * 10^{6}$ \\
\hline & ssGBLUP (30) & $11,373,208$ & 4929 & $3.269 * 10^{-6}$ & 3.934 & $1.203 * 10^{6}$ \\
\hline & ssSNPBLUP (10) & $11,627,330$ & 5001 & $3.270 * 10^{-6}$ & 3.921 & $1.199 * 10^{6}$ \\
\hline & ssSNPBLUP (30) & $11,627,330$ & 4937 & $3.269 * 10^{-6}$ & 3.931 & $1.202 * 10^{6}$ \\
\hline \multirow[t]{4}{*}{ KAR } & ssGBLUP (10) & $26,709,604$ & 3902 & $2.882 * 10^{-6}$ & 5.062 & $1.757 * 10^{6}$ \\
\hline & ssGBLUP (30) & $26,709,604$ & 3968 & $2.379 * 10^{-6}$ & 5.063 & $2.128 * 10^{6}$ \\
\hline & ssSNPBLUP (10) & $26,861,584$ & 4037 & $2.811 * 10^{-6}$ & 5.062 & $1.801 * 10^{6}$ \\
\hline & ssSNPBLUP (30) & $26,861,584$ & 3988 & $2.366 * 10^{-6}$ & 5.063 & $2.140 * 10^{6}$ \\
\hline \multirow[t]{2}{*}{ LVD } & ssGBLUP (10) & $96,688,714$ & 5431 & $6.248 * 10^{-6}$ & 8.635 & $1.382 * 10^{6}$ \\
\hline & ssSNPBLUP (10) & $96,916,684$ & 5888 & $5.312 * 10^{-6}$ & 8.935 & $1.682 * 10^{6}$ \\
\hline \multirow[t]{4}{*}{ LVD + block } & ssGBLUP (10) & $96,688,714$ & 1761 & $1.672 * 10^{-5}$ & 4.160 & $2.488 * 10^{5}$ \\
\hline & ssGBLUP (30) & $96,688,714$ & 1959 & $1.295 * 10^{-5}$ & 4.161 & $3.212 * 10^{5}$ \\
\hline & ssSNPBLUP (10) & $96,916,684$ & 2542 & $1.140 * 10^{-5}$ & 6.201 & $5.441 * 10^{5}$ \\
\hline & ssSNPBLUP (30) & $96,916,684$ & 2336 & $1.260 * 10^{-5}$ & 5.686 & $4.513 * 10^{5}$ \\
\hline
\end{tabular}

a Percentage of variance (due to additive genetic effects) explained by residual polygenic effects

b $\kappa=$ Effective spectral condition number of the preconditioned coefficient matrix 

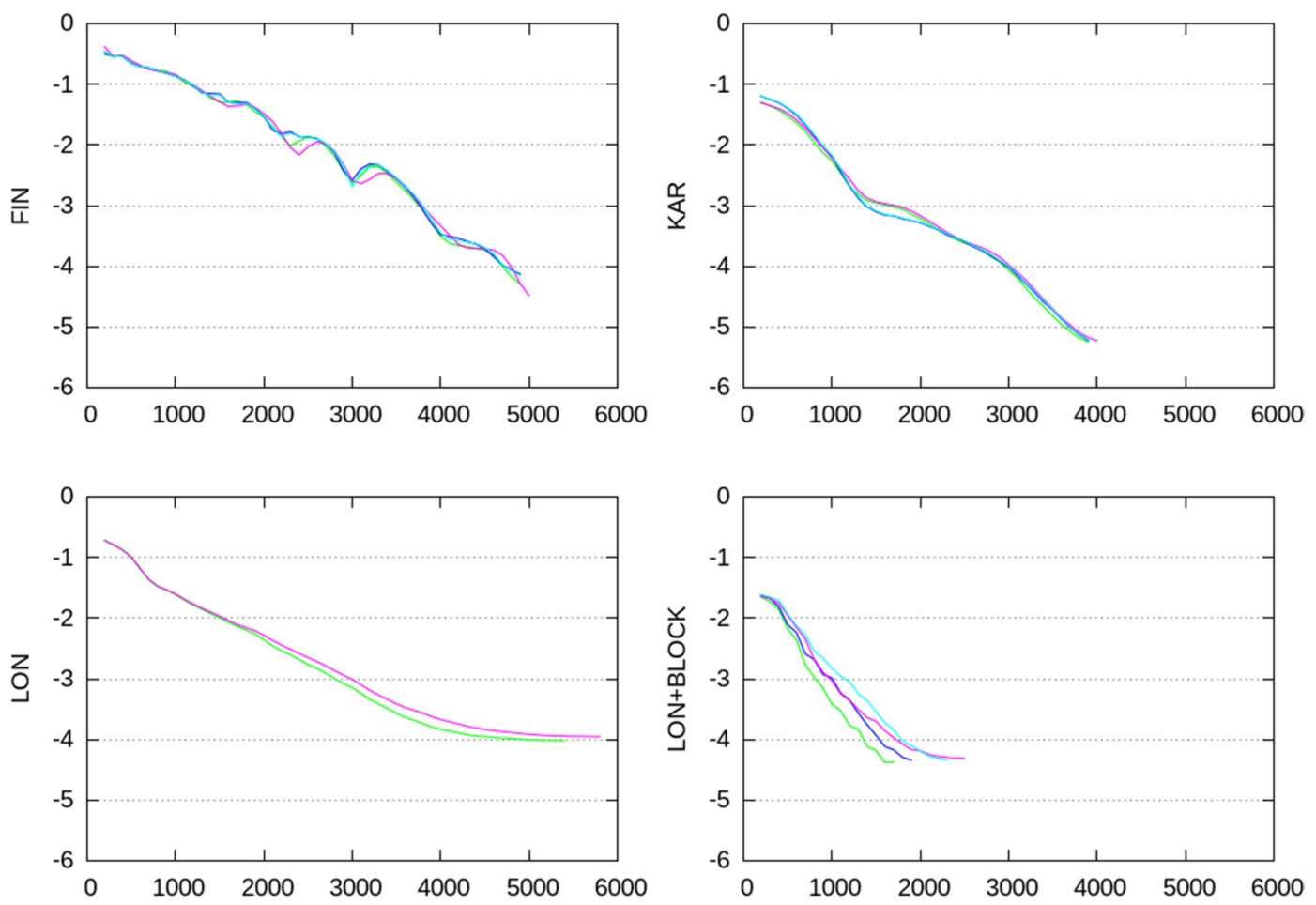

$\operatorname{ssGBLUP(10)}$

ssGBLUP(30)

- ssSNPBLUP(10)

SSSNPBLUP(30)

Fig. 2 Relative errors in the solutions for the different evaluations. Relative errors in the solutions are depicted for ssGBLUP with a proportion of residual polygenic variance equal to $10 \%$, for ssGBLUP with a proportion of residual polygenic variance equal to $30 \%$, for ssSNPBLUP with a proportion of residual polygenic variance equal to 10\%, and sSSNPBLUP with a proportion of residual polygenic variance equal to $30 \%$

eigendecomposition of a tridiagonal matrix of size equal to the number of iterations and computed following Eq. 2.3 in Kaasschieter [21].

The smallest Ritz value was used to estimate $\mu_{1}$, needed for the termination criterion $\mathrm{CK}$, and the ratio of the largest and smallest Ritz values was used to approximate $\kappa\left(\tilde{\mathbf{M}}^{-1} \mathbf{C}\right)$, needed for the termination criterion CM. Our initial analyses showed that the termination criteria $\mathrm{CK}$ and $\mathrm{CM}$ could result in stopping the iterative process too soon (i.e. before a desired level of accuracy of the solutions is achieved), because the smallest Ritz values computed at the beginning of the iterative process are poor estimates of the smallest active eigenvalues of the preconditioned coefficient matrix. Therefore, starting values for $\mu_{1}$ and $\kappa\left(\tilde{\mathbf{M}}^{-1} \mathbf{C}\right)$ were provided to the PCG method. These starting values for $\mu_{1}\left(\kappa\left(\tilde{\mathbf{M}}^{-1} \mathbf{C}\right)\right)$ were replaced by the Ritz value-based estimates when they became larger (smaller) than their corresponding estimates. Based on previous experiences, the starting values used for $\mu_{1}$ $\left(\kappa\left(\tilde{\mathbf{M}}^{-1} \mathbf{C}\right)\right)$ were set to $10^{-6}\left(10^{6}\right)$ for FIN, $10^{-5}$ $\left(1.5 * 10^{6}\right)$ for KAR, $10^{-5}\left(10^{6}\right)$ for $\mathrm{LON}$, and $2 * 10^{-5}$ $\left(10^{5}\right)$ for LON + block.

The solution vector was saved in a binary file every 100-th iteration starting at iteration 200. After termination, using the manufactured solutions as true solutions, the relative error in $\mathbf{x}, e_{r, i}$, was computed every 100-th iteration. The termination criteria CR and CD, as well as $\frac{\left\|\tilde{\mathbf{M}}^{-1} \mathbf{r}_{i}\right\|}{\left\|\hat{\mathbf{x}}_{i}\right\|}$ and $\frac{\left\|\tilde{\mathbf{M}}^{-1} \mathbf{r}_{i}\right\|}{\left\|\tilde{\mathbf{M}}^{-1} \mathbf{b}\right\|}$, were computed at each iteration. After the last PCG iteration was finished, the largest and smallest Ritz values were computed. The termination criteria $\mathrm{CK}$ and $\mathrm{CM}$ were then retrospectively computed for all iterations, with $\mu_{1}$ being approximated by the smallest Ritz value, and $\kappa\left(\tilde{\mathbf{M}}^{-1} \mathbf{C}\right)$ by the ratio of the largest and smallest Ritz values. The termination criterion $\mathrm{CR}$ for ssSNPBLUP was also computed by excluding equations for SNP effects.

Finally, from a practical point of view, a relative error in the solution $\leq 5 . * 10^{-3}$ might not be required in routine evaluations, and the iterative process could be stopped sooner. We investigated this assumption by determining the number of iterations needed to achieve a maximal absolute difference between intermediate and true 

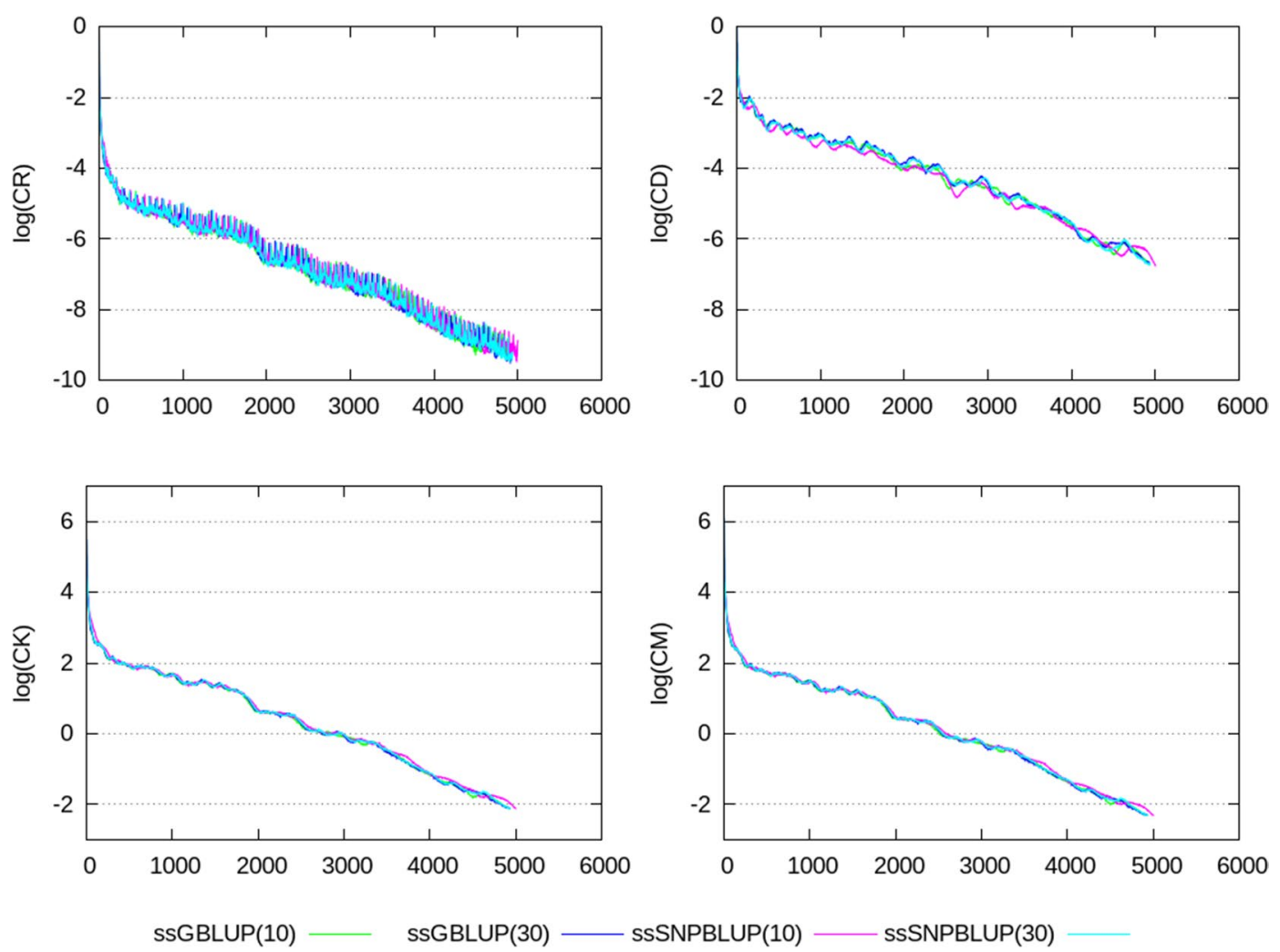

Fig. 3 Termination criteria for the FIN data set. Termination criteria are depicted for SSGBLUP with a proportion of residual polygenic variance equal to $10 \%$, for ssGBLUP with a proportion of residual polygenic variance equal to $30 \%$, for ssSNPBLUP with a proportion of residual polygenic variance equal to $10 \%$, and ssSNPBLUP with a proportion of residual polygenic variance equal to $30 \%$

genetic effects (or Legendre polynomials) lower than 1\% of a genetic standard deviation for all the traits. The number of iterations needed to achieve a Pearson correlation greater than 0.99990 between intermediate and true genetic effects (or Legendre polynomials) for each trait separately was also determined.

\section{Results}

\section{Ritz values and effective spectral condition numbers}

The Ritz values computed at different iterations are depicted in Fig. 1. For the four evaluations, the largest Ritz values varied between 3.9 and 8.9, and are well estimated within less than 200 iterations (Fig. 1; Table 1). Furthermore, within each of the four evaluations, the largest Ritz values of equivalent ssGBLUP and ssSNPBLUP were almost equal. Regarding the smallest Ritz values, their estimates follow a similar pattern for ssGBLUP and ssSNPBLUP. Therefore, they are of similar order for each of the four evaluations, and varied between $10^{-5}$ and $10^{-6}$ after termination (Fig. 1; Table 1). In all cases, both the largest and the smallest Ritz values for ssGBLUP and ssSNPBLUP do not seem to be influenced by the proportion of variance assigned to the residual polygenic effects. Finally, similar extremal Ritz values associated with different ssGBLUP and ssSNPBLUP resulted similar estimates of effective spectral condition numbers for these linear systems (i.e. all around $10^{6}$; Table 1).

\section{Termination criteria}

Termination criteria $e_{r}, \mathrm{CD}, \mathrm{CK}$, and $\mathrm{CM}$, shown in Figs. 2, 3, 4, 5, 6, show similar patterns for ssGBLUP and ssSNPBLUP for the four evaluations, and independently of the proportion of additive genetic variance assigned to the residual polygenic effects. This is not the case for the termination criterion $\mathrm{CR}$ that is associated with a pattern for ssSNPBLUP applied to KAR, LON, and LON + block a few folds higher than the corresponding pattern of CR for ssGBLUP. This behavior is not observed for FIN. Excluding the equations of the SNP effects for computing the termination criterion $\mathrm{CR}$ for sSSNPBLUP resulted in a pattern similar to the corresponding pattern for CR of ssGBLUP for all four evaluations (Fig. 7).

Maximal absolute differences between intermediate and true genetic effects (or Legendre polynomials) 

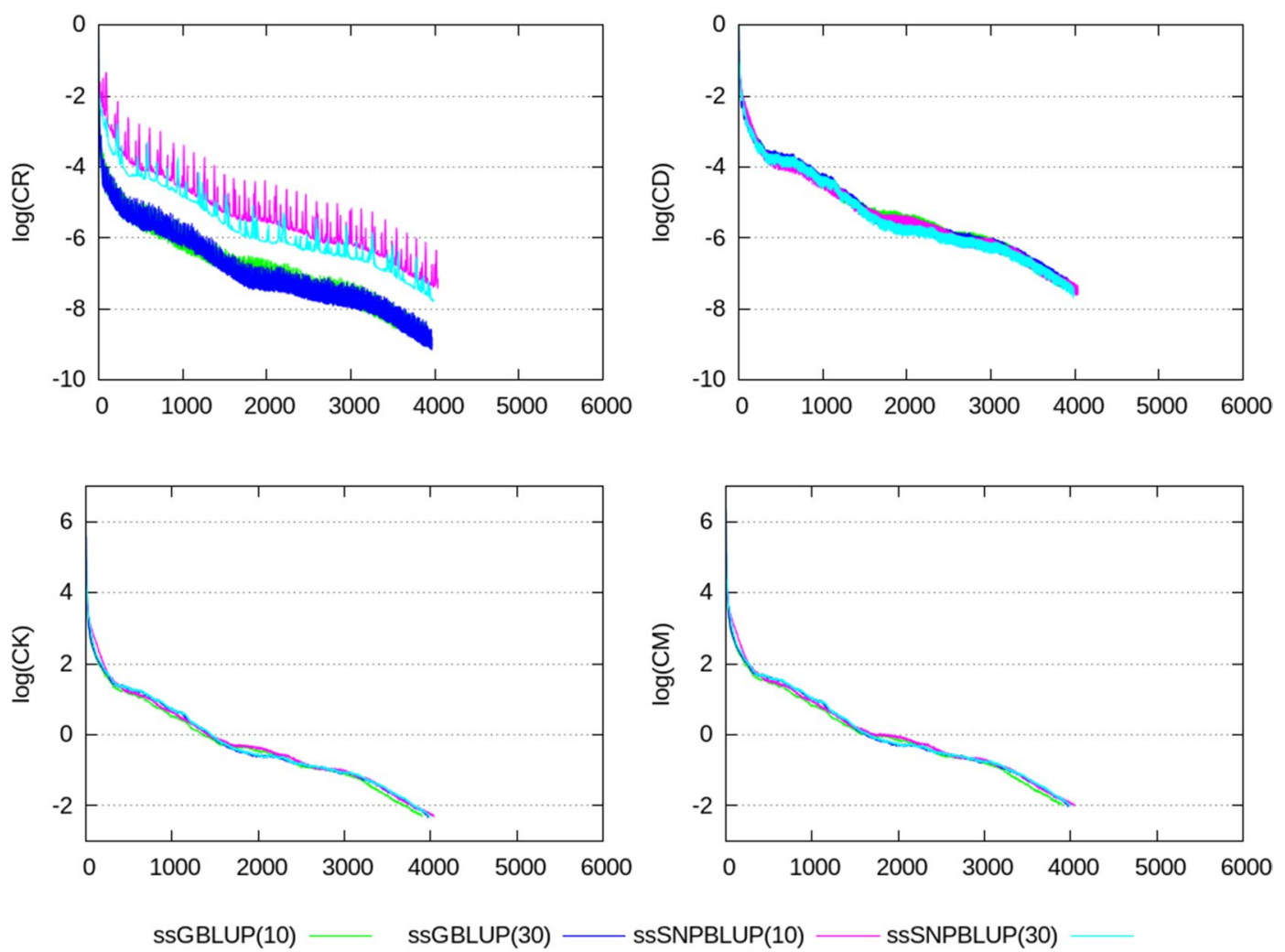

Fig. 4 Termination criteria for the KAR data set. Termination criteria are depicted for sSGBLUP with a proportion of residual polygenic variance equal to $10 \%$, for ssGBLUP with a proportion of residual polygenic variance equal to $30 \%$, for ssSNPBLUP with a proportion of residual polygenic variance equal to $10 \%$, and SsSNPBLUP with a proportion of residual polygenic variance equal to $30 \%$

lower than $1 \%$ of a genetic standard deviation for all traits were obtained for FIN after about $65 \%$ of the total number of iterations needed to satisfy a threshold of $5 * 10^{-3}$ for CK or CM, after around $35-47 \%$ for KAR, and after around $90 \%$ for $\mathrm{LON}$ and $\mathrm{LON}+$ block (Table 2). Even fewer iterations were needed to reach correlations between intermediate and true genetic effects (or Legendre polynomials) higher than 0.99990: around $53 \%$ of the total number of iterations for FIN, around $27 \%$ for KAR, and between 63 and $77 \%$ for LON and LON + block (Table 3).

\section{Discussion}

\section{Convergence behavior of the PCG method}

The rate of convergence of CG-based methods depends not only on the effective spectral condition number of the (preconditioned) coefficient matrix of the system being solved, but also on the distribution of eigenvalues of the (preconditioned) coefficient matrix [23, 29]. For the data sets used in this study, the PCG methods applied to ssGBLUP and ssSNPBLUP show an approximately linear convergence behavior in the logarithm of the relative error in the solution $\mathbf{x}, e_{r}$. Similar convergence behaviors can be also observed for other termination criteria in this study, as well as in other studies that used the PCG method to solve pedigree BLUP or ssGBLUP (e.g., [11, 12, 30]). These approximately linear convergence behaviors suggest that the spectra of the preconditioned coefficient matrices are composed of eigenvalues that are well distributed across the whole spectrum. This implies that these spectra have no, or only a few, isolated eigenvalues [23]. Such a well-distributed spectrum and linear convergence were also observed by Vandenplas et al. [9] who computed the entire spectrum of a preconditioned coefficient matrix for a small system of ssSNPBLUP developed by Mantysaari and Stranden [31]. Therefore, the rate of convergence of the PCG method applied to ssGBLUP or ssSNPBLUP mainly depends on the effective spectral condition number of the associated preconditioned coefficient matrix, and not on its associated distribution of eigenvalues. It follows that the PCG method applied to different evaluations (ssGBLUP or ssSNPBLUP) associated with similar effective spectral condition numbers should result in similar rates of convergence, convergence behavior, and number 

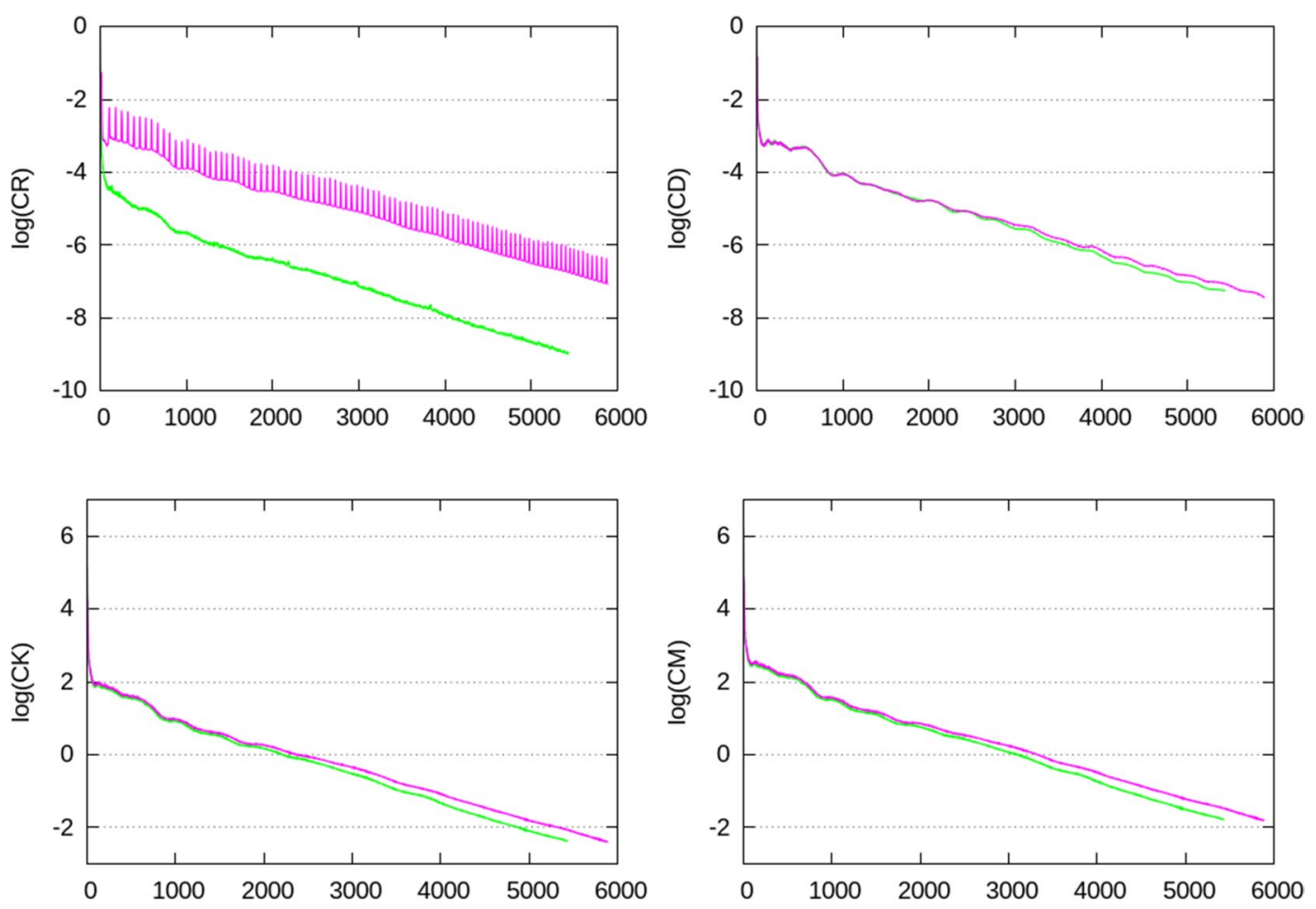

SSGBLUP(10)

SSSNPBLUP(10)

Fig. 5 Termination criteria for the LON data set when using a diagonal preconditioner. Termination criteria are depicted for ssGBLUP with a proportion of residual polygenic variance equal to 10\%, and SSSNPBLUP with a proportion of residual polygenic variance equal to $10 \%$

of iterations to meet a defined threshold of a specific termination criterion, provided that the termination criterion does not depend on the properties of the different systems, or considers them adequately. This was the case for the evaluations FIN, KAR, and LON. The different ssGBLUP and ssSNPBLUP evaluations for LON + block were associated to slightly different effective spectral condition numbers, resulting in different numbers of iterations to satisfy the same termination criterion.

\section{Termination criteria}

Four termination criteria were compared across different combinations of data sets and models. One of them, the termination criterion $\mathrm{CR}$, is related to the residual of the system, and shows different patterns when applied to ssGBLUP and ssSNPBLUP. These different behaviors can be explained by the errors in the estimates of the SNP effects. Indeed, excluding the SNP equations from the termination criterion CR for ssSNPBLUP resulted in patterns similar to those of ssGBLUP (Eq. (7); Fig. 7).
Using the termination criterion CR for ssSNPBLUP may lead to wrong conclusions, such as apparently nonaccurate solution estimates while they are actually accurate enough for practical use. Another wrong conclusion could be that the PCG method applied to ssSNPBLUP poorly converges in comparison to ssGBLUP.

The two termination criteria CK and CM are related to the solutions of the system, and show similar patterns when applied to ssGBLUP and ssSNPBLUP, independently of the data set or of the preconditioner used. Because the number of SNP effects is a small proportion of the total number of equations in large evaluations, and because the SNP effect estimates are relatively small in comparison to the estimates of the other solutions, convergence properties of the PCG method applied to ssGBLUP and ssSNPBLUP based on CK and CM are comparable. Based on these observations and on the properties of the four different termination criteria, the termination criteria $\mathrm{CK}$ and $\mathrm{CM}$ can be recommended for comparing convergence properties of different models with similar vectors of solutions. Based on our results, 

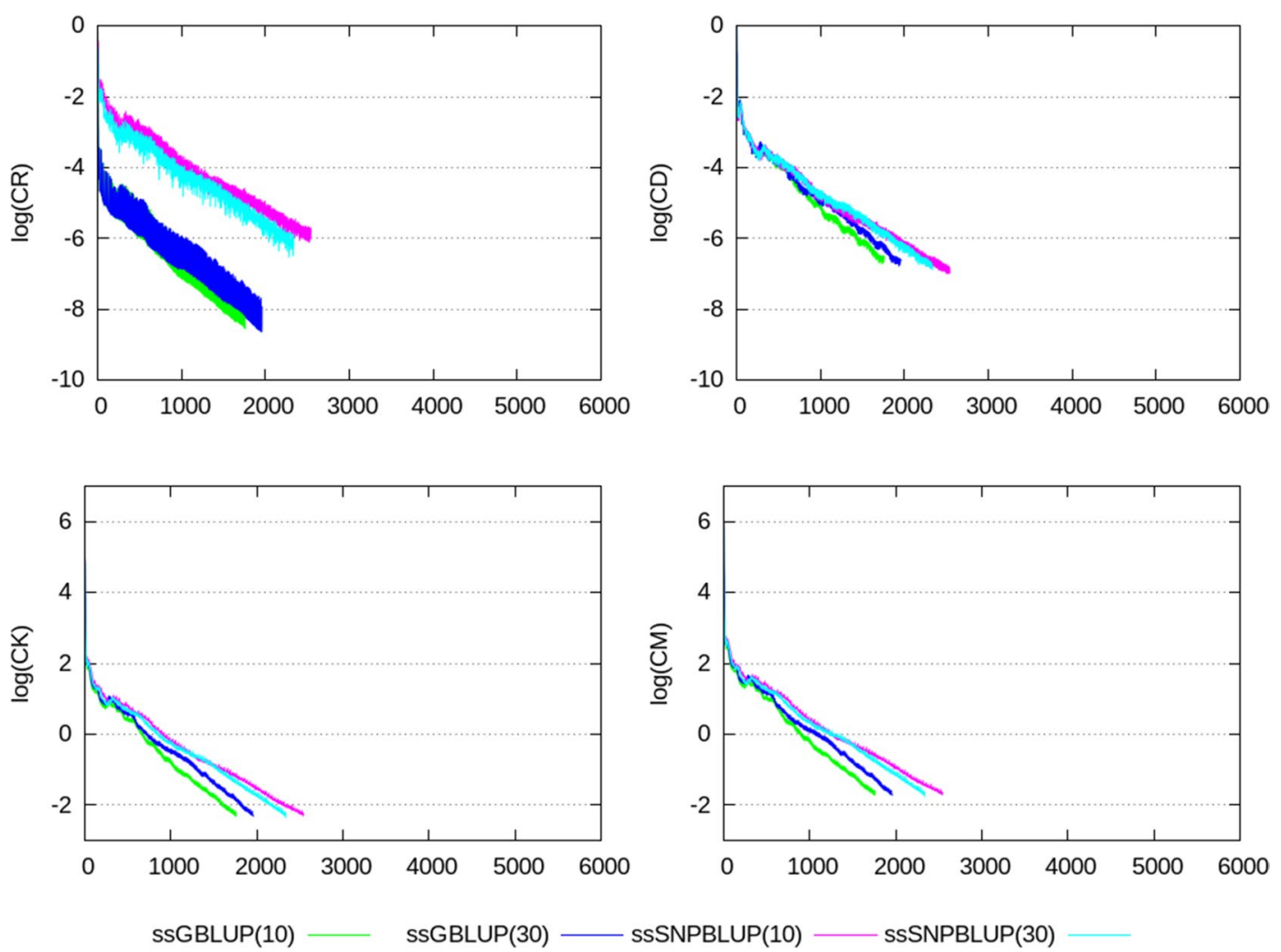

Fig. 6 Termination criteria for the LON data set when using a block-diagonal preconditioner. Termination criteria are depicted for sSGBLUP with a proportion of residual polygenic variance equal to 10\%, for ssGBLUP with a proportion of residual polygenic variance equal to $30 \%$, for ssSNPBLUP with a proportion of residual polygenic variance equal to 10\%, and SSSNPBLUP with a proportion of residual polygenic variance equal to $30 \%$

it seems that the termination criterion $\mathrm{CD}$ can be a good alternative to $\mathrm{CK}$ and $\mathrm{CM}$ for comparing convergence properties when no implementation of these two latter criteria is available. Finally, the termination criteria CK and $\mathrm{CM}$ are also recommended for use in routine evaluations, because they allow the users to specify a desired relative accuracy in $\mathbf{x}$.

\section{Threshold for termination criteria}

The threshold applied in this study for the termination criteria $\mathrm{CK}$ and $\mathrm{CM}$ was quite severe (i.e., $5 * 10^{-3}$ ). From a practical point of view, such an accuracy in the solutions is not required in routine evaluations, and the iterative process can be stopped sooner. For example, correlations between intermediate and true breeding values higher than 0.99990 were achieved within 27 and $77 \%$ of the total numbers of iterations needed to reach the severe threshold for CK and CM (Table 3). At this stage, Pearson correlations between intermediate and true SNP effects were all higher than 0.999. Based on the values obtained for the different termination criteria (Tables 2, 3), thresholds for both CK and CM around 0.2 for FIN, 2. for KAR, and 0.03 for LON and LON + block should therefore ensure enough accuracy in the solutions associated to the studied data sets for practical use. However, the adequacy of these thresholds should be checked and adapted for each evaluation.

\section{Implementation for $\mathrm{CK}$ and $\mathrm{CM}$}

In practice an estimate of the smallest active eigenvalue $\mu_{1}$ needed for the termination criterion CK, or of the effective spectral condition number needed for the termination criterion $\mathrm{CM}$, is unknown until the termination of the iterative process. Our initial analyses with an approach based on the Lanczos method to update an estimate of $\mu_{1}$ at each iteration (similarly to the approach proposed by Kaasschieter [21]), showed us that such an approach may lead to stop the iterative process too soon, i.e. before a desired level of accuracy of the solutions is achieved (results not shown). This undesirable behavior 

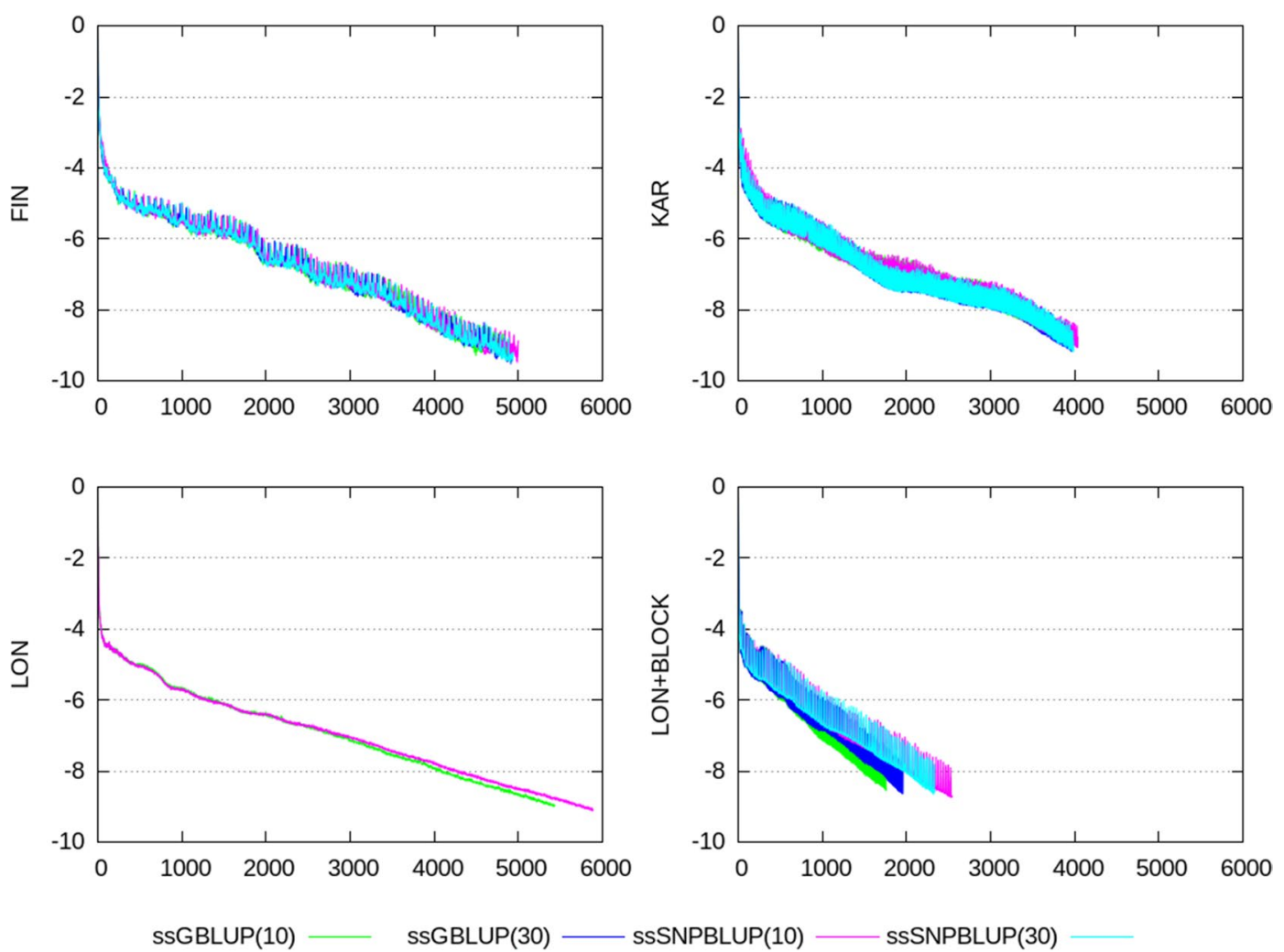

Fig. 7 Logarithm of termination criterion CR computed by excluding the SNP effects for different evaluations. Termination criterion CR is depicted for ssGBLUP with a proportion of residual polygenic variance equal to 10\%, for ssGBLUP with a proportion of residual polygenic variance equal to $30 \%$, for sSSNPBLUP with a proportion of residual polygenic variance equal to 10\%, and ssSNPBLUP with a proportion of residual polygenic variance equal to $30 \%$

was avoided by using conservative starting values for $\mu_{1}$ and $\kappa\left(\tilde{\mathbf{M}}^{-1} \mathbf{C}\right)$. These default values can be determined based on a previous analysis of a similar data set, or on previous experiences. Finally, the computation of the Ritz values at regular intervals (e.g., every 100-th iteration) allows to reduce its cost on the whole PCG process, even if this cost is relatively small. The main cost of the computation of the Ritz values is the eigendecomposition of a tridiagonal matrix of size equal to the number of iterations. In our analyses, each eigendecomposition took less than $1 \mathrm{~s}$ (wall clock time) until around 3000 iterations.

\section{Conclusions}

In this study, we proposed two termination criteria for the PCG algorithm that consider the properties of the system of equations being solved, and that can be related to the relative error in the solutions. Based on our implemented approaches and results, we showed that the PCG algorithms applied to ssSNPBLUP and ssGBLUP show similar convergence patterns, provided that the termination criterion does not depend on the properties of the different systems, or considers them adequately. We also showed that the Ritz values, that are approximations of the eigenvalues of the preconditioned coefficient matrix and that can be computed directly from the PCG outputs, are a good tool to better understand the convergence behavior of the PCG algorithm. 
Table 2 Number of iterations needed to reach a difference (for each trait) between intermediate and true estimates of genetic effects (or Legendre polynomials) lower than $1 \%$

\begin{tabular}{|c|c|c|c|c|c|c|c|}
\hline Evaluation & Model $^{a}$ & $\#$ Iterations $^{\mathrm{b}}$ & $e_{r}{ }^{c}$ & $C R$ & $C D$ & CK & CM \\
\hline \multirow[t]{4}{*}{ FIN } & ssGBLUP (10) & 3200 & $4.432 * 10^{-3}$ & $4.295 * 10^{-8}$ & $1.134 * 10^{-5}$ & 0.105 & 0.327 \\
\hline & ssGBLUP (30) & 3200 & $4.864 * 10^{-3}$ & $3.162 * 10^{-8}$ & $1.922 * 10^{-5}$ & 0.181 & 0.444 \\
\hline & ssSNPBLUP (10) & 3200 & $2.724 * 10^{-3}$ & $3.719 * 10^{-8}$ & $1.871 * 10^{-5}$ & 0.155 & 0.448 \\
\hline & ssSNPBLUP (30) & 3200 & $4.600 * 10^{-3}$ & $5.168 * 10^{-8}$ & $1.536 * 10^{-5}$ & 0.172 & 0.412 \\
\hline \multirow[t]{4}{*}{ KAR } & ssGBLUP (10) & 1800 & $8.745 * 10^{-4}$ & $6.520 * 10^{-8}$ & $5.535 * 10^{-6}$ & 0.431 & 0.877 \\
\hline & ssGBLUP (30) & 1400 & $9.561 * 10^{-4}$ & $7.483 * 10^{-1}$ & $8.794 * 10^{-6}$ & 1.163 & 2.372 \\
\hline & ssSNPBLUP (10) & 1900 & $8.180 * 10^{-4}$ & $6.845 * 10^{-6}$ & $4.194 * 10^{-6}$ & 0.444 & 0.905 \\
\hline & ssSNPBLUP (30) & 1500 & $8.181 * 10^{-4}$ & $4.504 * 10^{-6}$ & $6.830 * 10^{-6}$ & 0.802 & 1.636 \\
\hline \multirow[t]{2}{*}{ LON } & ssGBLUP (10) & 5200 & $9.709 * 10^{-5}$ & $1.590 * 10^{-9}$ & $6.699 * 10^{-8}$ & 0.006 & 0.023 \\
\hline & ssSNPBLUP (10) & 5600 & $1.126 * 10^{-4}$ & $6.966 * 10^{-7}$ & $5.583 * 10^{-8}$ & 0.006 & 0.025 \\
\hline \multirow[t]{4}{*}{ LON + block } & ssGBLUP (10) & 1600 & $4.178 * 10^{-5}$ & $1.868 * 10^{-8}$ & $5.976 * 10^{-7}$ & 0.010 & 0.039 \\
\hline & ssGBLUP (30) & 1800 & $5.085 * 10^{-5}$ & $5.054 * 10^{-9}$ & $5.041 * 10^{-7}$ & 0.011 & 0.042 \\
\hline & ssSNPBLUP (10) & 2000 & $6.559 * 10^{-5}$ & $4.742 * 10^{-6}$ & $7.822 * 10^{-7}$ & 0.021 & 0.083 \\
\hline & ssSNPBLUP (30) & 2100 & $5.314 * 10^{-5}$ & $1.372 * 10^{-6}$ & $4.656 * 10^{-7}$ & 0.013 & 0.052 \\
\hline
\end{tabular}

Values of termination criteria corresponding to the number of iterations are reported

${ }^{\text {a }}$ Percentage of variance (due to additive genetic effects) explained by residual polygenic effects

${ }^{b}$ The solutions were stored and evaluated every 100-th iteration

${ }^{\mathrm{c}} e_{r}=$ relative errors in the solutions

Table 3 Number of iterations needed to reach a Pearson correlation (for each trait) between intermediate and true estimates of genetic effects (or Legendre polynomials) greater than 0.99990

\begin{tabular}{|c|c|c|c|c|c|c|c|}
\hline Evaluation & Model $^{a}$ & \# Iterations ${ }^{b}$ & $e_{r}{ }^{c}$ & $C R$ & $C D$ & CK & CM \\
\hline \multirow[t]{4}{*}{ FIN } & ssGBLUP (10) & 2700 & $9.547 * 10^{-3}$ & $1.623 * 10^{-7}$ & $4.237 * 10^{-5}$ & 0.241 & 0.749 \\
\hline & ssGBLUP (30) & 2600 & $1.282 * 10^{-2}$ & $7.813 * 10^{-8}$ & $3.195 * 10^{-5}$ & 0.327 & 0.802 \\
\hline & sSSNPBLUP (10) & 2500 & $9.372 * 10^{-3}$ & $1.274 * 10^{-\prime}$ & $5.587 * 10^{-5}$ & 0.530 & 1.533 \\
\hline & ssSNPBLUP (30) & 2700 & $1.069 * 10^{-2}$ & $2.117 * 10^{-7}$ & $4.144 * 10^{-5}$ & 0.335 & 0.805 \\
\hline \multirow[t]{4}{*}{ KAR } & ssGBLUP (10) & 1100 & $3.460 * 10^{-3}$ & $3.293 * 10^{-7}$ & $3.967 * 10^{-5}$ & 2.595 & 5.284 \\
\hline & ssGBLUP (30) & 1100 & $3.675 * 10^{-3}$ & $1.063 * 10^{-6}$ & $2.904 * 10^{-5}$ & 4.096 & 8.354 \\
\hline & ssSNPBLUP (10) & 1100 & $4.115 * 10^{-3}$ & $1.851 * 10^{-5}$ & $1.683 * 10^{-5}$ & 2.737 & 5.572 \\
\hline & sSSNPBLUP (30) & 1100 & $4.039 * 10^{-3}$ & $1.135 * 10^{-5}$ & $2.435 * 10^{-5}$ & 4.189 & 8.544 \\
\hline \multirow[t]{2}{*}{ LON } & SSGBLUP (10) & 3900 & $1.607 * 10^{-4}$ & $1.540 * 10^{-8}$ & $6.789 * 10^{-7}$ & 0.060 & 0.235 \\
\hline & ssSNPBLUP (10) & 4100 & $1.974 * 10^{-4}$ & $1.849 * 10^{-6}$ & $5.344 * 10^{-7}$ & 0.069 & 0.267 \\
\hline \multirow[t]{4}{*}{ LON + block } & ssGBLUP (10) & 1300 & $1.476 * 10^{-4}$ & $2.273 * 10^{-8}$ & $2.121 * 10^{-6}$ & 0.040 & 0.154 \\
\hline & ssGBLUP (30) & 1500 & $1.177 * 10^{-4}$ & $1.987 * 10^{-8}$ & $2.003 * 10^{-6}$ & 0.043 & 0.165 \\
\hline & ssSNPBLUP (10) & 1600 & $1.393 * 10^{-4}$ & $1.549 * 10^{-5}$ & $2.839 * 10^{-6}$ & 0.073 & 0.282 \\
\hline & ssSNPBLUP (30) & 1800 & $9.499 * 10^{-5}$ & $5.961 * 10^{-6}$ & $1.352 * 10^{-6}$ & 0.039 & 0.153 \\
\hline
\end{tabular}

Values of termination criteria corresponding to the number of iterations are reported

${ }^{\text {a }}$ Percentage of variance (due to additive genetic effects) explained by residual polygenic effects

${ }^{b}$ The solutions were stored and evaluated every 100 -th iteration

${ }^{c} e_{r}=$ relative errors in the solutions 


\section{Supplementary Information}

The online version contains supplementary material available at https://doi. org/10.1186/s12711-021-00626-1.

Additional file 1. Derivation of the termination criterion CR.

Additional file 2. Derivation of the termination criterion CM.

Additional file 3. Relationship between the residual of the system of equations of sSGBLUPand sSSNPBLUP.

\section{Acknowledgements}

The use of the high-performance cluster was made possible by CAT-AgroFood (Shared Research Facilities Wageningen UR, Wageningen, the Netherlands).

\section{Authors' contributions}

JV conceived the study design, ran the tests, and wrote the programs and the first draft. JV and CV discussed and developed the theory. HE, MvP, and RB prepared data. MvP suggested the block-diagonal preconditioner for the LON analyses. All authors provided valuable insights throughout the writing process. All authors read and approved the final manuscript.

\section{Funding}

This study was financially supported by the Dutch Ministry of Economic Affairs (TKI Agri \& Food Project 16022) and the Breed4Food partners Cobb Europe (Colchester, Essex, United Kingdom), CRV (Arnhem, the Netherlands), Hendrix Genetics (Boxmeer, the Netherlands), and Topigs Norsvin (Helvoirt, the Netherlands).

\section{Declarations}

\section{Ethics approval and consent to participate}

The data used for this study were collected as part of routine data recording for a commercial breeding program. Samples collected for DNA extraction were only used for the breeding program. Data recording and sample collection were conducted strictly in line with the Dutch law on the protection of animals (Gezondheids- en welzijnswet voor dieren).

\section{Consent for publication}

Not applicable.

\section{Competing interests}

The authors declare that they have no competing interests.

\section{Author details}

${ }^{1}$ Animal Breeding and Genomics, Wageningen UR, P.O. Box 338, 6700 AH Wageningen, The Netherlands. ${ }^{2}$ CRV BV, Wassenaarweg, 20, 6843 NW Arnhem, The Netherlands. ${ }^{3}$ Topigs Norsvin, P.O. Box 43, 6640 AA Beuningen, The Netherlands. ${ }^{4}$ DIAM, TU Delft, Van Mourik Broekmanweg, 6, 2628 XE Delft, The Netherlands.

Received: 7 September 2020 Accepted: 25 March 2021 Published online: 09 April 2021

\section{References}

1. Legarra A, Christensen OF, Aguilar I, Misztal I. Single step, a general approach for genomic selection. Livest Sci. 2014;166:54-65.

2. Christensen OF, Lund MS. Genomic prediction when some animals are not genotyped. Genet Sel Evol. 2010;42:2.

3. Legarra A, Aguilar I, Misztal I. A relationship matrix including full pedigree and genomic information. J Dairy Sci. 2009;92:4656-63.

4. Fernando RL, Dekkers JC, Garrick DJ. A class of Bayesian methods to combine large numbers of genotyped and non-genotyped animals for whole-genome analyses. Genet Sel Evol. 2014;46:50.

5. Fernando RL, Cheng H, Golden BL, Garrick DJ. Computational strategies for alternative single-step Bayesian regression models with large numbers of genotyped and non-genotyped animals. Genet Sel Evol. 2016;48:96

6. Liu Z, Goddard M, Reinhardt F, Reents R. A single-step genomic model with direct estimation of marker effects. J Dairy Sci. 2014;97:5833-50.

7. Strandén I, Lidauer M. Solving large mixed linear models using preconditioned conjugate gradient iteration. J Dairy Sci. 1999;82:2779-87.

8. Taskinen M, Mäntysaari EA, Strandén I. Single-step SNP-BLUP with onthe-fly imputed genotypes and residual polygenic effects. Genet Sel Evol. 2017:49:36.

9. Vandenplas J, Eding H, Calus MPL, Vuik C. Deflated preconditioned conjugate gradient method for solving single-step BLUP models efficiently. Genet Sel Evol. 2018;50:51.

10. Vandenplas J, Calus MPL, Eding H, Vuik C. A second-level diagonal preconditioner for single-step SNPBLUP. Genet Sel Evol. 2019;51:30.

11. Lidauer M, Strandén I, Mäntysaari EA, Pösö J, Kettunen A. Solving large test-day models by iteration on data and preconditioned conjugate gradient. J Dairy Sci. 1999;82:2788-96.

12. Tsuruta S, Misztal I, Stranden I. Use of the preconditioned conjugate gradient algorithm as a generic solver for mixed-model equations in animal breeding applications. J Anim Sci. 2001;79:1166-72.

13. Meyer K. Technical note: a successive over-relaxation preconditioner to solve mixed model equations for genetic evaluation. J Anim Sci. 2016:94:4530-5

14. Aguilar I, Misztal I, Johnson DL, Legarra A, Tsuruta S, Lawlor TJ. Hot topic: a unified approach to utilize phenotypic, full pedigree, and genomic information for genetic evaluation of Holstein final score. J Dairy Sci. 2010;93:743-52.

15. Gengler N, Nieuwhof G, Konstantinov K, Goddard ME. Alternative single-step type genomic prediction equations. In: Proceedings of the $63 r d$ annual meeting of the European Association for Animal Production: 27-31 August 2012; Bratislava. 2012

16. Mäntysaari EA, Evans RD, Strandén I. Efficient single-step genomic evaluation for a multibreed beef cattle population having many genotyped animals. J Anim Sci. 2017;95:4728-37.

17. Saad Y. Iterative methods for sparse linear systems. Other titles in applied mathematics. 2nd ed. Philadelphia: Society for Industrial and Applied Mathematics; 2003.

18. Barrett R, Berry MW, Chan TF, Demmel J, Donato J, Dongarra J, et al. Templates for the solution of linear systems: building blocks for iterative methods, vol. 43. Philadelphia: Society of Industrial and Applied Mathematics; 1994.

19. Axelsson O, Kaporin I. Error norm estimation and stopping criteria in preconditioned conjugate gradient iterations. Numer Linear Algebra Appl. 2001;8:265-86

20. Nabben $R$, Vuik C. A comparison of deflation and the balancing preconditioner. SIAM J Sci Comput. 2006;27:1742-59.

21. Kaasschieter EF. A practical termination criterion for the conjugate gradient method. BIT Numer Math. 1988;28:308-22.

22. Vuik C, Segal A, Meijerink JA. An efficient preconditioned CG method for the solution of a class of layered problems with extreme contrasts in the coefficients. J Comput Phys. 1999:152:385-403.

23. Sluis Avd, Vorst HAvd. The rate of convergence of conjugate gradients. Numer Math. 1986:48:543-60.

24. Statistical indicators, E16: breeding value-Temperament during milking. https://cooperatiecrv-be6.kxcdn.com/wp-content/uploads/2020/04/E 16-Gedrag-April-2020-Engels.pdf. Accessed 29 Mar 2021.

25. Statistical indicators, E-15: Breeding value milking speed. https://coope ratiecrv-be6.kxcdn.com/wp-content/uploads/2020/04/E 15-Melksnelhe id-April-2020-Engels.pdf. Accessed 29 Mar 2021.

26. CRV Animal Evaluation Unit. E-19: breeding values for longevity (LON); 2019. https://cooperatiecrv-be6.kxcdn.com/wp-content/uploads/2019/ 08/E 19-Longevity aug2019 eng.pdf. Accessed 29 Mar 2021.

27. van Pelt M, de Jong G, Veerkamp RF. Improving the genetic evaluation for longevity in the Netherlands. Interbull Bull. 2017;51:33-7.

28. Vandenplas J, Eding H, Bosmans M, Calus MPL. Computational strategies for the preconditioned conjugate gradient method applied to SSSNPBLUP, with an application to a multivariate maternal model. Genet Sel Evol. 2020;52:24

29. Strakoš Z. On the real convergence rate of the conjugate gradient method. Linear Algebra Appl. 1991;154-156:535-49. 
30. Pocrnic I, Lourenco DAL, Bradford HL, Chen CY, Misztal I. Technical note: impact of pedigree depth on convergence of single-step genomic BLUP in a purebred swine population. J Anim Sci. 2017;95:3391-5.

31. Mäntysaari EA, Strandén I. Single-step genomic evaluation with many more genotyped animals. In: Proceedings of the 67th annual meeting of the European Association for Animal Production: 29 August-2 September 2016; Belfast. 2016

\section{Publisher's Note}

Springer Nature remains neutral with regard to jurisdictional claims in published maps and institutional affiliations.
Ready to submit your research? Choose BMC and benefit from:

- fast, convenient online submission

- thorough peer review by experienced researchers in your field

- rapid publication on acceptance

- support for research data, including large and complex data types

- gold Open Access which fosters wider collaboration and increased citations

- maximum visibility for your research: over $100 \mathrm{M}$ website views per year

At BMC, research is always in progress.

Learn more biomedcentral.com/submissions 